\title{
Practices and pitfalls in inferring neural representations
}

\author{
Vencislav Popov $^{\mathrm{a}, \mathrm{b}, *}, 1$, Markus Ostarek ${ }^{\mathrm{c}, 1}$, Caitlin Tenison ${ }^{\mathrm{a}, \mathrm{b}}$ \\ a Department of Psychology, Carnegie Mellon University, 5000 Forbes Avenue, Baker Hall, 15289, Pittsburgh, PA, USA \\ ${ }^{\mathrm{b}}$ Center for the Neural Basis of Cognition, 4400 Fifth Ave, 15213, Pittsburgh, PA, USA \\ ${ }^{\mathrm{c}}$ Max Planck Institute for Psycholinguistics, PO Box 310, 6500, AH Nijmegen, The Netherlands
}

\section{A R T I C L E I N F O}

\section{Keywords:}

Representation

fMRI

Encoding models

Multivariate pattern analysis

\begin{abstract}
A B S T R A C T
A key challenge for cognitive neuroscience is deciphering the representational schemes of the brain. Stimulusfeature-based encoding models are becoming increasingly popular for inferring the dimensions of neural representational spaces from stimulus-feature spaces. We argue that such inferences are not always valid because successful prediction can occur even if the two representational spaces use different, but correlated, representational schemes. We support this claim with three simulations in which we achieved high prediction accuracy despite systematic differences in the geometries and dimensions of the underlying representations. Detailed analysis of the encoding models' predictions showed systematic deviations from ground-truth, indicating that high prediction accuracy is insufficient for making representational inferences. This fallacy applies to the prediction of actual neural patterns from stimulus-feature spaces and we urge caution in inferring the nature of the neural code from such methods. We discuss ways to overcome these inferential limitations, including model comparison, absolute model performance, visualization techniques and attentional modulation.
\end{abstract}

\section{Introduction}

A key challenge for cognitive neuroscience is to understand the neural code that underlies the encoding and representation of sensory, motor, spatial, emotional, semantic and other types of information. To decipher the representational schemes of the brain, researchers often employ neuroimaging techniques such as functional magnetic resonance imaging (fMRI). fMRI measures the blood oxygenation level-dependent (BOLD) activation in the brain that is elicited when participants engage with different stimuli. The neural representation underlying each stimulus is assumed to have measurable but complex effects on the BOLD activation patterns. In order to understand what those patterns of activity can tell us about how the brain processes and represents information, researchers have used various analytical tools such as univariate subtraction methods, multivariate pattern (MVP) classification, representational similarity analysis (RSA) and, recently, explicit stimulus-feature-based encoding and decoding models (for reviews, see Davis and Poldrack, 2013, Haxby et al., 2014, or Naselaris et al., 2011). Despite their differences, all of these methods have the same goal - to quantify how changes in task conditions and the properties of the stimuli relate to changes in BOLD activation and vice versa. One way in which these methods differ is in how they achieve that mapping and in what inferences they allow us to draw.

In this article, we review some of the known inferential limitations of existing fMRI analysis methods and we highlight an often-overlooked issue in interpreting results from stimulus-feature-based encoding and decoding models. The latter are steadily becoming the de facto gold standard for investigating neural representational spaces (Haxby et al., 2014; Naselaris and Kay, 2015). Using simulated data with known representational schemes, we demonstrate the limitations of these analysis methods. These simulations, the accompanying code and the text also provide a useful guide for using encoding models to understand neural representations.

\section{Univariate vs. multivariate analysis}

Two of the main questions that any fMRI analysis technique attempts to answer are 1) where information is represented/processed and 2) what the nature of those representations is (Davis and Poldrack, 2013). Across the fMRI analysis techniques we review below it becomes apparent that understanding how information is represented is more difficult than understanding where it is represented or processed.

\footnotetext{
* Corresponding author. Carnegie Mellon University, 5000, Forbes Avenue, Baker Hall, 345G, Pittsburgh, PA, USA.

E-mail address: vencislav.popov@gmail.com (V. Popov).

1 VP and MO contributed equally and should be considered co-first authors.
} 
The localization and the representational questions are related, but distinct. This difference is most clearly exemplified in univariate subtraction analyses. Before the advent of the more advanced techniques we review below, the standard approach for analyzing fMRI data involved comparing how activity in a single voxel or averaged activity in a contiguous area of voxels differs between task conditions or stimuli (see Fig. 1). Researchers have used these univariate subtraction methods successfully to understand the relative engagement of certain brain areas in specific tasks. For example, the observation that remembered stimuli cause greater hippocampal activation during their encoding compared to forgotten stimuli, has corroborated conclusions from lesion studies about the role of the medial temporal lobe in memory formation (Wagner et al., 1998).

Unfortunately, the coarse nature of this method precludes finegrained inferences about the underlying representational content and computations that give rise to the observed BOLD signal. Univariate methods assume that representational or processing differences between stimuli can be observed in individual voxels, and they ignore any relationships between different voxels (Davis and Poldrack, 2013). By ignoring the possibility that information might be represented in a distributed manner across voxels, the assumptions underlying univariate subtraction methods limit their use in understanding neural representations. In addition, these methods cannot tell us whether changes in activation are due to representational preferences, processing differences, or attentional variation among conditions (Coutanche, 2013).

In contrast, multivoxel pattern analysis (MVPA) techniques overcome these limitations by looking at how various categories of stimuli or task conditions lead to combinatorial differences (i.e. MVP classification) or similarities (i.e. representational similarity analysis, RSA, see Fig. 2) in distributed patterns of activity over multiple voxels. These methods have become popular partly because they allow researchers to relate brain states to informational content. This can be highly useful for real-world applications, such as brain-computer interfaces (Lotte et al., 2007) and the diagnosis of diseases (Ewers et al., 2011). More importantly for the present paper, MVPA methods make it possible to study neural representational spaces with greater sensitivity and resolution than univariate approaches. For example, a seminal study by Haxby et al. (2001) found that visual object categories can be classified based on the pattern of activation that their exemplars elicited in the ventral temporal cortex. The classification was successful despite the lack of overall activation differences in that region. Similar methods have been used to show that concepts have language-invariant representations in the anterior temporal lobe (Correia et al., 2014), that very similar visual scenes can be discriminated in the hippocampus (Bonnici et al., 2012) and that during their retrieval from memory, the shape, color and identity of visual objects can be differentially decoded across several cortical areas (Coutanche and Thompson-Schill, 2015).

Despite early enthusiasm that MVPA methods could be used to understand the structure of the neural code and the nature of the underlying representations (Norman et al., 2006), conventional MVP classification and RSA techniques share one of the same fundamental inferential limitations of univariate methods. Researchers can use successful classification or careful inspection of confusions/similarity matrices to learn that some relevant information about the stimulus class is present in the population of analyzed voxels, thus answering the where question. However, these methods cannot identify unambiguously exactly what that information is (i.e., the stimulus category, category-related features, etc), or how it is represented and organized (Naselaris and Kay, 2015; Poldrack, 2011; Tong and Pratte, 2012). Neural data is correlational, which means that many different properties of the stimuli might lead to successful classification of the stimulus category, the task condition, or the brain state in question. For example, successfully categorizing whether a word represents an animate or an inanimate object does not necessarily mean that the region of interest encodes that category distinction. There are many differences between animate and inanimate objects, such as differences in their sensory and functional features (Farah and McClelland, 1991) that could be responsible for the successful classification.

In a similar argument, Ritchie et al. (2017) recently pointed out that the main reason behind this limitation is that linear classifiers are "informationally greedy": they are likely to pick up any information that distinguishes the to-be-classified categories and it is therefore often impossible to know what that information is. This uncertainty can lead to disagreements between researchers looking at similar data. For instance, it remains unclear whether the orientation of a grating can be decoded

\section{Univariate MRI}

\section{MR signal in region $X$}
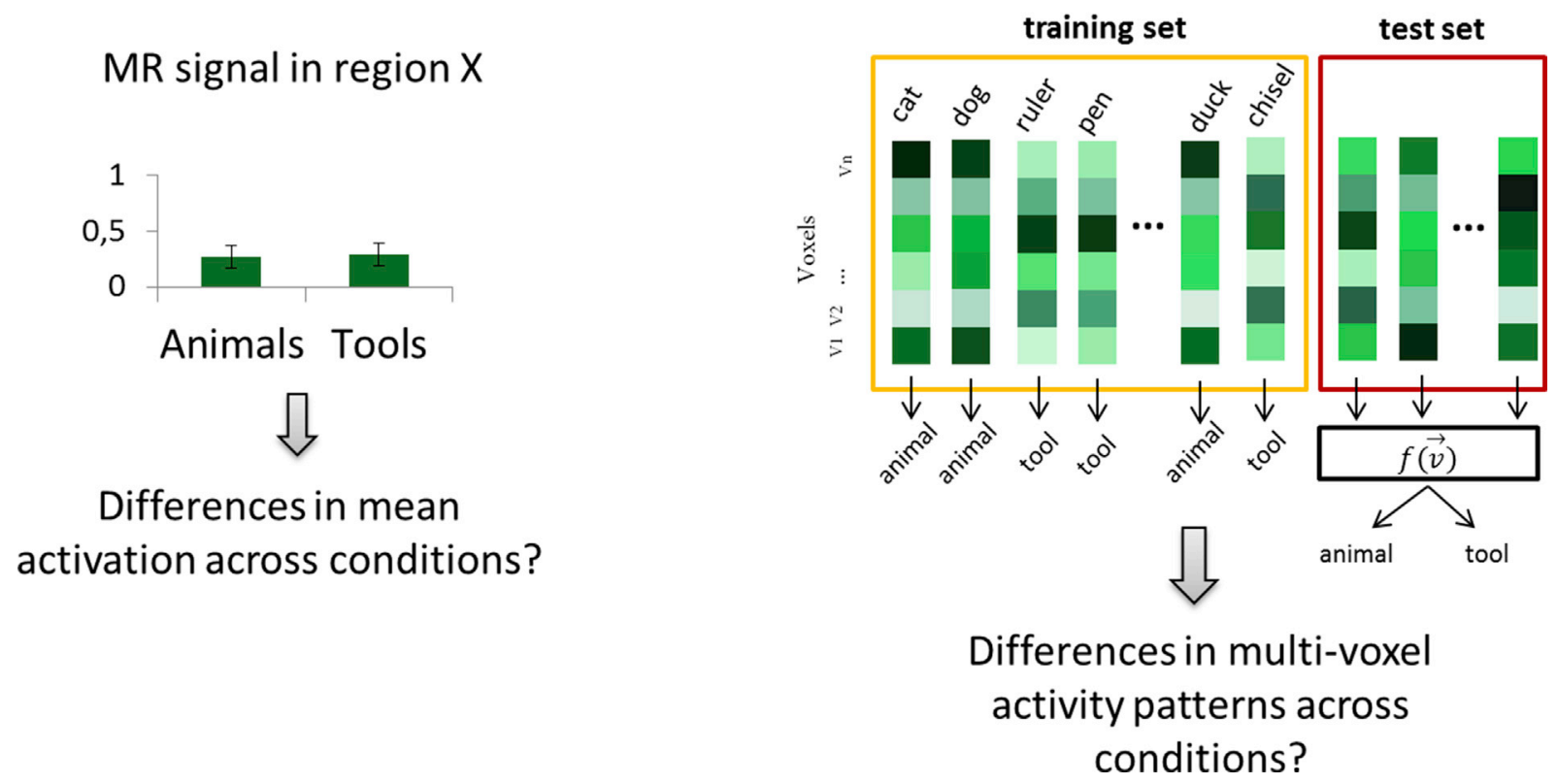

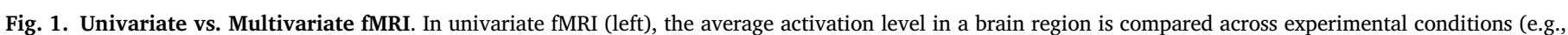

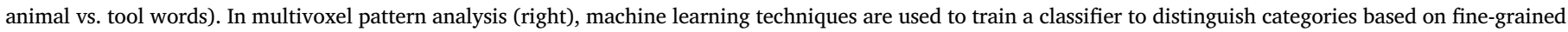
activation patterns (yellow box) and its classification performance is evaluated on a separate test set (red box). 


\section{fMRI activation patterns}

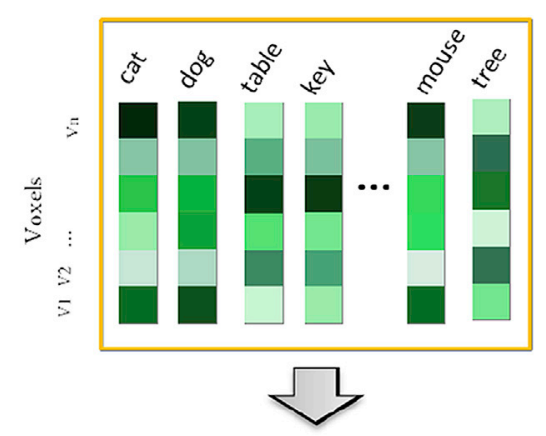

fMRI pattern dissimilarity

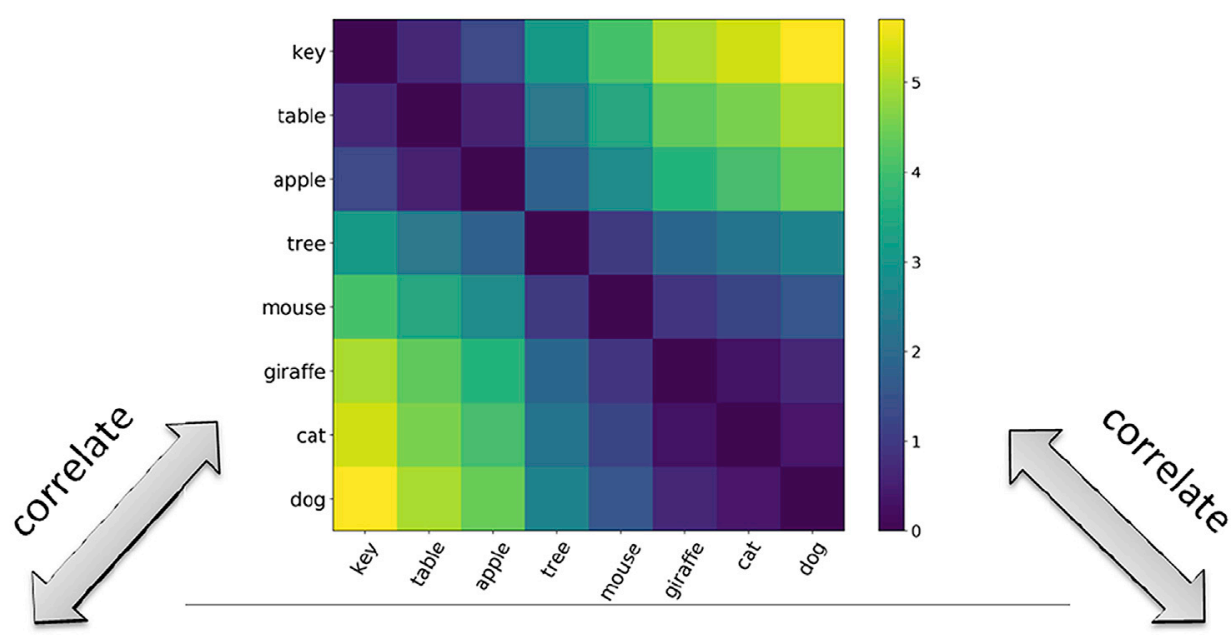

Behavioural size ratings

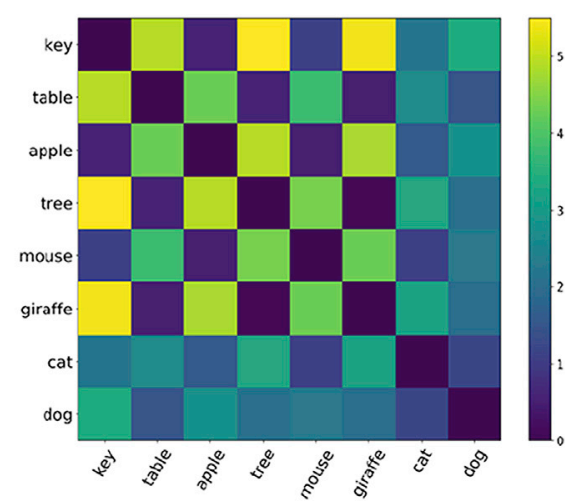

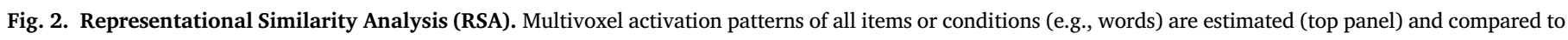

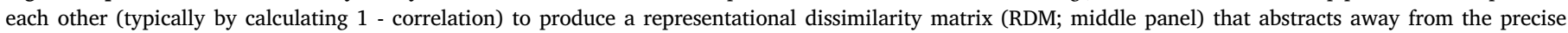

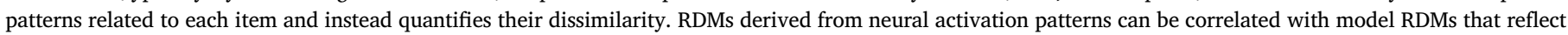

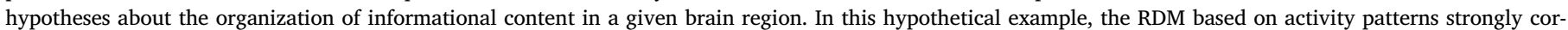
relates with the RDM derived from behavioral ratings of animacy, but not with the RDM derived from behavioral ratings of size.

from visual cortex because imperfect sampling of orientation-selective columns leads to hyperacuity (Kamitani \& Tong, 2005), because of radial biases in the retinotopic map (Freeman et al., 2011), or because of edge-related activity (Carlson, 2014). Ritchie et al. (2017) further suggest that relating classifier performance to behavior only partly remedies the problem as "a brain region might carry information which is reliably correlated with the information that is actually used, but which is not itself used in behavior".

Another limitation of conventional MVP classifiers is that they cannot generalize and predict behavioral responses to novel types of stimuli or task conditions. An MVP classifier is usually trained on stimuli that are tokens from several types. The relationship between these tokens and types impacts the generality of the classifier. The stimuli tokens might be different category exemplars, and the classifier is trained to predict the type of category to which they belong. In this case, the classifier can only be used to predict category membership of items that belong to one of the categories on which it was trained. For instance, if one trains a classifier to predict the color of objects and trains it on yellow and orange objects 
(Coutanche and Thompson-Schill, 2015), one will not be able to predict the color of novel objects that are green. Alternatively, the tokens might be multiple presentations of the same word in different modalities or languages and the types are the unique words themselves. Even though the classifier in this example could be used to predict exemplars in novel languages or modalities, it is again restricted only to exemplars of the words on which it was trained in the first place. In general, while the tokens being tested might be novel, they can be successfully classified only if they are exemplars of a type that has already been trained on.

This methodological limitation is important - just as understanding how the decimal system represents numbers allows people to understand and manipulate numbers they have never seen before, understanding how a neural representational system works should allow researchers to use the neural pattern associated with novel stimuli to predict their identity, even if those stimuli are not exemplars of the types on which a particular model was trained on.

\section{Encoding models}

To overcome these limitations many researchers are turning to a novel analysis method that is known by a few different names - voxelwise modelling (Naselaris and Kay, 2015), stimulus-model based encoding and decoding (Haxby et al., 2014), voxel-based encoding and decoding models (Naselaris et al., 2011), and forward models (Brouwer and Heeger, 2009; Fernandino et al., 2016). For simplicity, we will refer to this class of methods as encoding models. ${ }^{2}$

In contrast to MVP classifiers, which assign one of the trained category labels to a novel stimulus token, encoding models can predict the identity of novel types of stimuli. They achieve that by attempting to predict the neural activity for a set of simpler features into which the stimuli can be decomposed (see Fig. 3). In a seminal study, Mitchell et al. (2008) predicted the neural activity associated with individual novel words based only on the activation of other words. They decomposed each word into a vector of weights on 25 sensory-motor semantic features (verbs such as "eat", "taste", "run", "fear", etc.). The weights were estimated from co-occurrence statistics of the word with each verb feature in a large corpus. They trained a classifier to predict the neural activity associated with each constituent feature of a training set of words, which resulted in separate neural activation maps for each feature. Neural activity for novel test words was then predicted highly accurately as a linear combination of the semantic feature activation maps weighted by the association of the word with each feature. Based on these results, Mitchell et al. (2008) concluded that the neural representation of concrete nouns might be based on sensory-motor features.

Similar approaches have been used to predict the neural response to novel natural images using Gabor filter features (Kay et al., 2008), to novel colors based on color tuning curve features (Brouwer and Heeger, 2009), to novel music clips based on acoustic timbre features (Casey et al., 2012), to natural sounds based on frequency, spectral and temporal modulations (Santoro et al., 2014), to novel faces based on a PCA decomposition of face features (Lee and Kuhl, 2016), to novel words based on subjective sensory-motor ratings (Fernandino et al., 2016). The motivating question behind many of these studies has been about the nature of the representations used by the brain in encoding the experimental stimuli, and the results are often cited as evidence that the neural representation is based on the constituent features of the stimuli used in the model.

In general, most encoding models use the following analysis procedure (see also Fig. 3):

\footnotetext{
${ }^{2}$ Encoding usually refers to the prediction of neural patterns for the purpose of understanding the neural representational space, while decoding refers to the reconstruction of an experienced stimulus from neural data (Naselaris et al., 2011).
}

1 Specify a set of features that hypothetically underlie the representation of a stimulus set in the brain

2 Decompose a set of stimuli into vectors of weights for each feature

3 Select a region of interest (ROI) in the brain from which to analyze neural activation

4 Train a model to predict activity in each voxel for a training set of stimuli, using the weights of their features as predictors

5 Derive activation pattern maps (e.g. regression coefficients) associated with each feature

6 .Predict neural activity in the ROI for novel stimuli, based on their feature weights and the activation pattern maps for each feature

7 Compare predicted neural activity for each novel stimulus with their observed neural activity and derive a measure of fit and accuracy

Encoding models attempt to map a stimulus feature representational space, where each feature is a separate dimension, and each stimulus is a point in that space, to a neural activation space, where each voxel or each neuron is a separate dimension, and the activation pattern elicited by each stimulus is a point in that space (Kriegeskorte and Kievit, 2013).

\section{What can we infer about neural representations?}

Encoding models have grown in popularity due to their many advantages over more classical classification approaches. While there is little doubt that these models are a useful and powerful analytic tool, their growing use requires us to carefully consider what we can and cannot conclude on the basis of above-chance prediction of neural activity. What can a successful mapping between a stimulus feature space and a neural activation space tell us about the nature of the representation used by the brain? A common inference in some of these studies is that if you can predict the identity of novel stimuli based on that mapping, then the neural representation is likely based on the feature set used by the model. Put formally, the inferential claim goes as follows:

- We can represent certain stimuli as a combination of lower-level features

- We can show that it is possible to predict the neural pattern caused by a novel stimulus in brain area A from an encoding model based on these features

- Therefore, brain area A encodes those features and uses a representational scheme based on them.

This claim has been made to different degrees both in theoretical and methodological papers reviewing the approach (e.g., Haxby et al., 2014; Naselaris and Kay, 2015; Naselaris et al., 2011; Norman et al., 2006; Ritchie et al., 2017; Tong and Pratte, 2012), as well as in empirical studies that use it to address representational questions (Fernandino et al., 2016; Kay et al., 2008; Mitchell et al., 2008; Santoro et al., 2014; although some are more cautionary, e.g. Lee and Kuhl, 2016). While some authors have carefully restrained their discussion to the practical benefits of encoding models (Haxby et al., 2014; Davis and Poldrack, 2013), the interpretative benefits of encoding models over MVP classification are expressed strongly by many others (e.g., Naselaris and Kay, 2015; Ritchie et al., 2017).

A useful illustration of this inference in practice comes from a recent study by Fernandino et al. (2016). The authors wanted to understand how conceptual information is represented in a set of higher-order non-modality-specific brain regions in the General Semantic Network (GSN; Binder et al., 2009). An encoding model based on subjective ratings for 5 sensory-motor features ("color", "motion", "sound", "shape", "action") of training words was used to predict neural activation patterns related to novel individual words. The model successfully predicted above chance the brain activity patterns for concrete words in the semantic network regions ( $61 \%$ mean accuracy), but not in a set of control regions associated with visual word form processing. Based on these finding, Fernandino et al. (2016) suggested that "the brain represents concepts as multimodal 


\section{Training set}

Model is trained to predict voxelwise activity for each stimulus by learning activation maps for each constituent feature of the stimuli
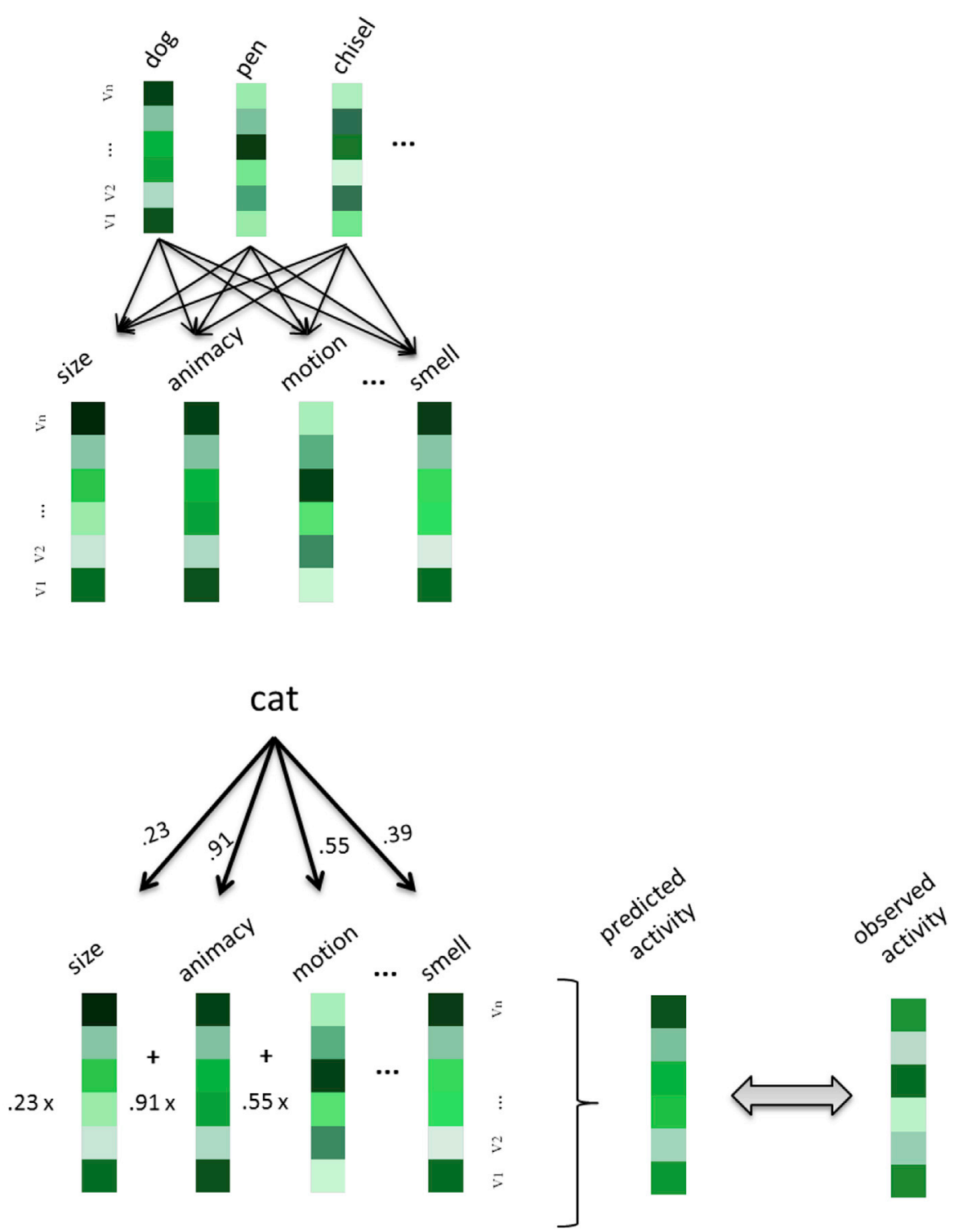

\section{Test set}

Model predicts activity related to novel items by linear combination of the feature activation maps weighted by the items' associations with the features

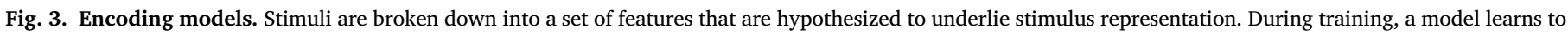

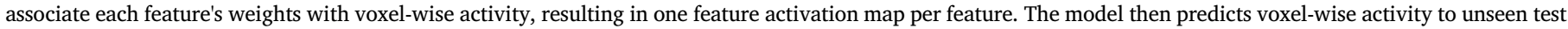

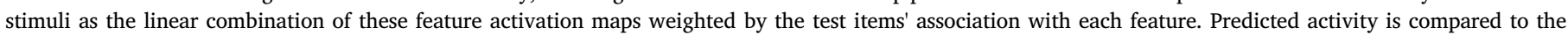
actually observed activity per voxel to quantify prediction accuracy.

combinations of sensory and motor representations" and that "heteromodal areas involved in semantic processing encode information about the relative importance of different sensory-motor attributes of concepts, possibly by storing particular combinations of sensory and motor features" (p. 9763).

Putting aside the fact that $61 \%$ prediction rank accuracy is quite low, albeit above chance, ${ }^{3}$ the more important issue is that this inference is not formally valid. We need to consider what the data would have looked like if the underlying neural representation was actually different (Mahon, 2015). In this example, the above-chance prediction of conceptual identity in the GSN based on an encoding model of sensory-motor features does not necessitate the representational format in the GSN to be sensory-motor in nature. The results might be obtained even if the GSN

\footnotetext{
${ }^{3}$ In praxis, success of an encoding model is often defined as above-chance prediction and thus treated like an all-or-nothing phenomenon. This is in part because absolute prediction values are difficult to interpret (Hebart and Baker, 2017). However, attaining above-chance prediction does not justify strong claims about the nature of neural representations.
}

uses amodal representations, as long as the representational space used by the GSN and the sensory-motor features are correlated. This correlation could be driven by a non-arbitrary mapping between representations in the GSN and sensory-motor features.

To illustrate, let us assume that the GSN instead encodes word cooccurrence statistics. As co-occurrence statistics correlate with sensorymotor feature ratings, it would be possible to predict GSN activity patterns above-chance based on these features, even though they are not driving the activity patterns. Thus, while we can rule out the possibility that conceptual representations in heteromodal areas bear an arbitrary relation to sensory-motor features, as has been argued by some proponents of symbolic systems (Fodor \& Pylyshyn, 1988), we cannot conclude that the GSN encodes multimodal sensory-motor information on the basis of Fernandino et al. (2016) results. At most, we can say that the subjective sensory-motor relevance ratings that Fernandino et al. (2016) gathered for each concept capture some information represented in the GSN, but not whether this information is sensory-motor in nature.

This issue is akin to the affirming the consequent fallacy. It is the case that if the brain uses a specific representational scheme, an encoding model 
Table 1

Limitations of representational analyses for fMRI.

\begin{tabular}{|c|c|c|c|}
\hline Limitation & Univariate & MVP & $\begin{array}{l}\text { Encoding } \\
\text { models }\end{array}$ \\
\hline 1. Ignores distributed information & + & - & - \\
\hline $\begin{array}{l}\text { 2. Cannot predict activity for novel types of } \\
\text { stimuli }\end{array}$ & + & + & - \\
\hline $\begin{array}{l}\text { 3. Sensitive to correlated sources of } \\
\text { information }\end{array}$ & + & + & + \\
\hline
\end{tabular}

that implements those feature will likely be able to predict neural activation fairly well. However, we cannot reverse this claim - successful prediction of neural activity based of an encoding model does not guarantee that the model in question is correct. ${ }^{4}$ We argue that information in one representational system might be decoded or predicted above-chance based on features from another, even if they use different representational schemes, as long as there is at least a partially systematic mapping between them, which causes their dimensions to be correlated. This limitation is shared with univariate and MVP approaches (see Table 1). There are at least three ways in which mappings between representational systems can be made and successful prediction can occur in two of those cases.

\section{Types of mappings}

\section{Arbitrary mappings between representations}

First, items from two representational systems might be related in an entirely arbitrary way, which results in no shared variance in their dimensions. For example, the meaning of words is mostly unrelated to their orthographic features, ${ }^{5}$ and the geographic locations of countries are not predictive of their names, etc. More generally, consider two unordered sets of items, $A=\left\{A_{1}, A_{2}, \ldots, A_{n}\right\}$ and $B=\left\{B_{1}, B_{2}, \ldots, B_{n}\right\}$ An arbitrary mapping between these two sets exists when the mapping from a specific item in set $\mathrm{A}$ to a corresponding item in set $\mathrm{B}$ is unrelated to the mappings between the remaining items in the two sets. In the context of encoding models and the brain, predicting the identity of novel items from one set would be impossible based on a feature model from the other set, if these two sets are not correlated.

\section{Sets that use the same representational scheme}

In contrast, a successful prediction can occur if the two sets use the same representational scheme. Consider the set of multi-digit numbers in the decimal system, $A=\{10,11, \ldots, 427, \ldots\}$, and the set of 10 digits in the decimal system, $B=\{0,1,2,3,4,5,6,7,8,9\}$. These sets use the same representational scheme to represent quantities (the decimal system), and there is a systematic linear mapping from the features (the digits), to the multi-digit numbers, such that:

$\overline{d_{n} d_{n-1} \ldots d_{1} d_{0}}=\sum_{i=0}^{n}\left(d_{i} \times 10^{i}\right)$

$3491=3 \times 1000+4 \times 100+9 \times 10+1 \times 1$

When we have such systematic mappings between systems that use the same representational scheme, knowing the mapping function allows us to decompose any item from set A as a combination of features from set B. An example of such a mapping would be Fernandino et al. (2016) suggestion that the General Semantic Network encodes multimodal

\footnotetext{
${ }^{4}$ This problem is similar, but not identical, to the problem of reverse inference (Poldrack, 2006).

5 Whereas a minor degree of systematicity does seem to exist in this domain (e.g., Monaghan et al., 2014), word meanings cannot be systematically predicted based on their orthography and vice versa.
}

Table 2

Examples of studies that use feature encoding models.

\begin{tabular}{|c|c|c|c|}
\hline Source & Item & Features & Response vector \\
\hline $\begin{array}{l}\text { Mitchell } \\
\text { et al. } \\
\text { (2008) }\end{array}$ & $\begin{array}{l}\text { Concrete } \\
\text { words } \\
(\operatorname{dog})\end{array}$ & $\begin{array}{l}\text { Co-occurrence } \\
\text { statistics with } \\
25 \text { sensory- } \\
\text { motor verbs }\end{array}$ & $\begin{array}{l}\text { Pattern of activation in all cortical } \\
\text { voxels }\end{array}$ \\
\hline $\begin{array}{l}\text { Fernandino } \\
\text { et al. } \\
\text { (2016) }\end{array}$ & $\begin{array}{l}\text { Concrete } \\
\text { words } \\
(\operatorname{dog})\end{array}$ & $\begin{array}{l}5 \text { sensory- } \\
\text { motor } \\
\text { relevance } \\
\text { ratings }\end{array}$ & $\begin{array}{l}\text { Pattern of activation in the GSN } \\
\text { (Binder et al., 2009) }\end{array}$ \\
\hline $\begin{array}{l}\text { Simulation } \\
\quad 1\end{array}$ & $\begin{array}{l}\text { Numbers } \\
\text { (3497) }\end{array}$ & $\begin{array}{l}5 \text { decimal digits } \\
{\left[\begin{array}{lllll}0 & 3 & 4 & 9 & 7\end{array}\right]}\end{array}$ & 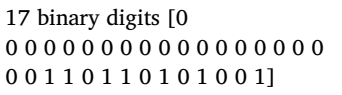 \\
\hline $\begin{array}{l}\text { Simulation } \\
\quad 2\end{array}$ & $\begin{array}{l}\text { Colors } \\
\text { (skyblue) }\end{array}$ & $\begin{array}{l}\text { RGB values, } \\
{[135206235]}\end{array}$ & HSV values $[197,42.6,92.2]$ \\
\hline $\begin{array}{l}\text { Simulation } \\
\quad 3\end{array}$ & $\begin{array}{l}\text { Colors } \\
\text { (skyblue) }\end{array}$ & $\begin{array}{l}\text { HSV values } \\
{[197,42.6,} \\
92.2]\end{array}$ & $\begin{array}{l}\text { Population code based on } 5 \\
\text { preferred values on each RGB } \\
\text { dimension }\end{array}$ \\
\hline
\end{tabular}

combinations of sensory-motor features by integrating information from modality-specific sensory-motor areas. If this were true, then you could predict the neural pattern of novel items from their featural representations, which is what that study found as well.

\section{Sets that use different but systematically related representational schemes}

However, there is an alternative, which would also allow you to make a successful prediction from encoding models due to shared variance in nonequivalent representational spaces. Two sets can use different representational schemes, while at the same time maintaining a systematic mapping between themselves. That systematicity results in correlated dimensions and it allows us to predict the mapping of any one pair of items from knowledge of the mapping function. Within the context of conceptual representations in the brain, higher-level heteromodal areas might use a representational code that is different from the one used by sensory-motor cortices, but the representational dimensions in the two systems might be correlated.

For a simplified example, consider the relation between the decimal and the binary systems for representing numeric values. A binary represented value can be transformed into a decimal number by applying the following formula:

$$
\begin{aligned}
& \left(\overline{d_{n} d_{n-1} \ldots d_{0}}\right)_{2} \rightarrow\left(\sum_{i=0}^{n}\left(d_{i} \times 2^{i}\right)\right)_{10} \\
& 10011_{2} \rightarrow 1 \times 2^{4}+0 \times 2^{3}+0 \times 2^{2}+1 \times 2^{1}+1 \times 2^{0}=16_{10}+2_{10}+1_{10} \\
& \quad=19_{10}
\end{aligned}
$$

Clearly, there is a systematic but non-linear mapping between the decimal and the binary system, and yet, these two systems use different codes to represent numbers. If our argument is correct then it should be possible to predict above chance the binary representation of a number based on a decimal feature encoding model. Below we present a simulation that achieves this by applying the encoding model approach often used in neuroimaging studies. Within the simulation, binary vectors are analogous to voxel activation patterns, and the encoding model is based on decimal representations (Table 2).

\section{Simulation 1: predicting binary representations with a decimal feature encoding model}

${ }^{6}$ As detailed previously, encoding models predict stimulus identity

\footnotetext{
${ }^{6}$ The code for all simulations is available at https://github.com/venpopov/ inferential_pitfalls.
} 
from brain activation by modelling the relationship between the constituent features of the training stimuli and their corresponding BOLD activation in a group of voxels. Then they use that relationship to estimate the expected neural activation patterns for novel test items based on their feature representations. The predicted activation pattern for each stimulus is compared to the observed patterns for all test stimuli. For the following simulation, let us consider the numbers from 0 to 99999 as our stimulus set. They can be decomposed into 5-dimensional feature vectors where each feature is a decimal digit (e.g., 3497 can be decomposed as [0 $\left.\begin{array}{lll}3 & 4 & 9\end{array}\right]$. These features can be considered analogous to the 5 sensorymotor relevance ratings of words used by Fernandino et al. (2016) or to the co-occurrence statistics with sensory-motor verbs used by Mitchell et al. (2008). Further, let us consider the binary representation numbers as 17-dimensional vectors (e.g. [0 000000000000000000000000011

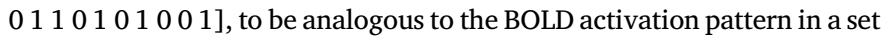
of 17 voxels in an ROI under investigation. The correspondence between these patterns and actual neuroimaging studies using this approach is demonstrated in Table 2.

We trained an encoding model to predict the binary activation pattern for a given number, based on its 5-dimensional decimal feature representation. We performed a 10-fold cross-validation. The modelling followed 4 steps: 1) splitting the stimuli into a training (90\%) and a test $(10 \%)$ set, 2) fitting multiple linear regression models on the training set with the 17 binary features as response variables, and the 5 decimal features as predictors, 3) calculating predicted activation pattern (predicted maps, PMs) for each test item from its decimal features and the multivariate regression model, 4) comparing the PMs with the actual binary patterns for all test items (observed maps, OMs). In the comparison stage, we computed the Euclidean distance between each PM and the OMs for all test items, and we calculated the percentile rank of the similarity between the PM and the OM of each item. For example, if the PM for the number 29782 were most similar to the OM for that number, then the percentile rank for it would be $10000 / 10000=1$. However, if it were more similar to the OMs of 1000 other items, then its percentile rank would be $9000 / 10000=0.9$.

The encoding model successfully predicted above chance the binary representation of untrained items based only on their decimal features $\left(\mathrm{M}_{\text {accuracy }}=0.7, \mathrm{SD}=0.24, \mathrm{p}<.0001\right.$, Wilcoxon signed rank test $)$. Since by definition binary and decimal number systems use different representational schemes, we cannot conclude that the representation of binary numbers encodes decimal features. By analogy, successfully predicting patterns of neural activation based on a stimulus feature space, cannot be used to infer that the brain encodes information about these features or that its neural representational space is organized along the dimensions of that feature space.

Simulation 2: predicting a color represented in one color space with an encoding model in another color space

Another way to illustrate how two correlated, but distinct representational spaces can be predicted from one another, is to use color representational spaces. Any color can be uniquely defined as a point in a three-dimensional space. There exist numerous color spaces, and in some of them the dimensions reflect three distinct colors that can be mixed in different proportions to produce any other color (e.g., RGB, CMY), while in other color spaces the dimensions reflect attributes such as hue, saturation and lightness (e.g., HSL, HSV). RGB and HSV are both threedimensional spaces; however, their dimensions are related non-linearly (see Fig. 4). As a result, their representational geometry is different, and for example, one color can be equally distant from two others in RGB space, while it can be closer to one of them in HSV space. Despite this, the position of colors within those two spaces are systematically but nonlinearly related, and there exist a number of algorithms to convert RGB representations to HSV and vice versa (for a primer on color spaces and color space conversions, see Ford and Roberts, 1998).

The goal of the following simulation was to demonstrate that we can successfully predict the three valued HSV representation of novel colors, by training an RGB encoding model on a subset of randomly sampled colors. For the stimuli, we used 1000 colors by selecting 10 equally spaced values on each RGB dimensions. Thus, the stimulus features of each color were its three RGB values. The response vectors in this case were the corresponding three HSV values for each color. The procedure was otherwise identical to the one in Simulation 1. We performed a 10fold cross-validation. Similar to Simulation 1, the encoding model based on RGB color representations was able to predict the HSV color representations of untrained colors quite well (mean rank accuracy 0.82 , $\mathrm{SD}=0.22, \mathrm{p}<.0001)$.

The current simulation allows us to illustrate how such a high predictive accuracy in and of itself might not provide enough information to judge how well the encoding model reflects the neural representational space. For each item, we can directly compare the predicted color from the encoding model with the actual color of the stimulus. In Fig. 5, we show the "observed" ground truth and the predicted color for each test item. Each panel shows hues for different combinations of saturation and
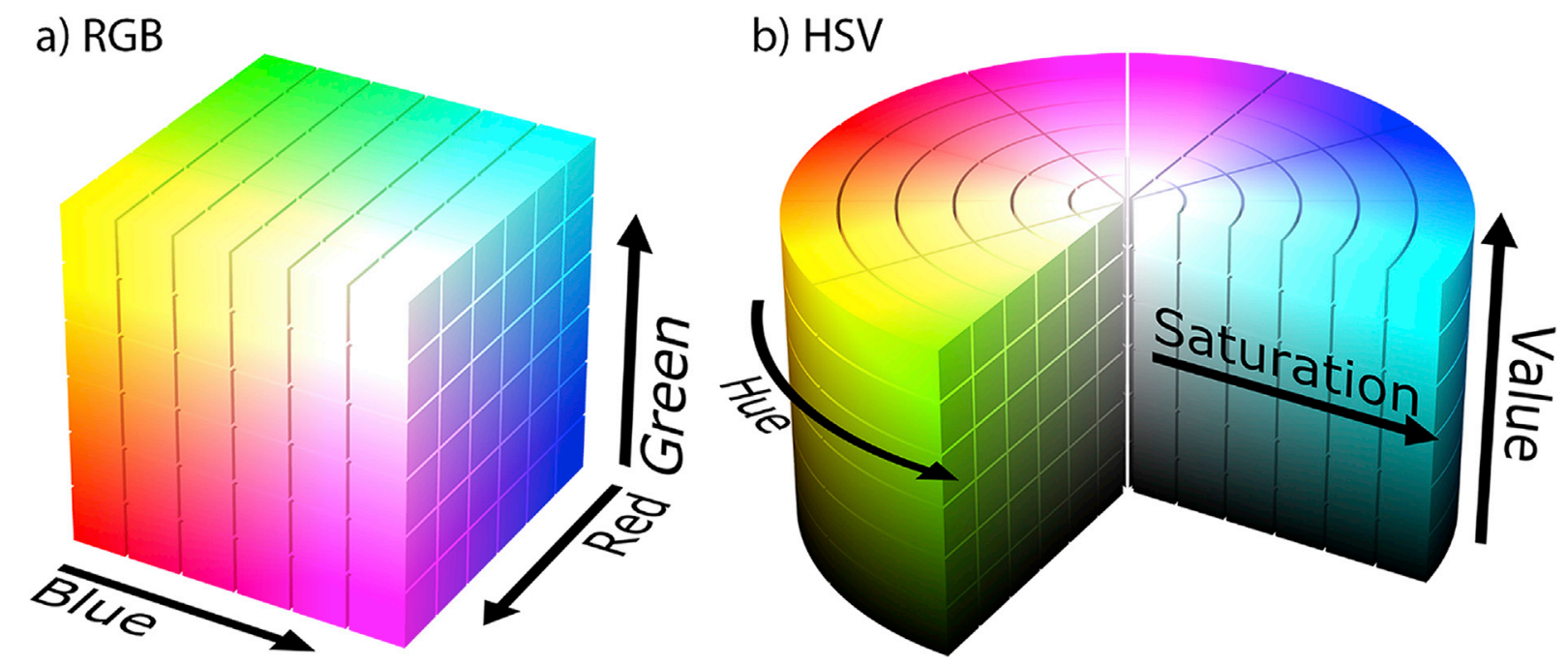

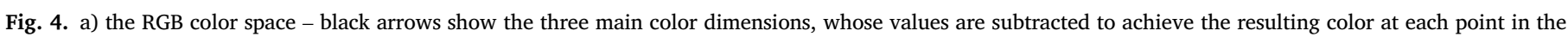

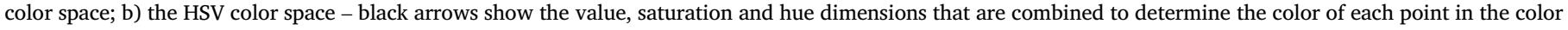
space. Images by Michael Horvath, available under Creative Commons Attribution-Share Alike 3.0 Unported license. 


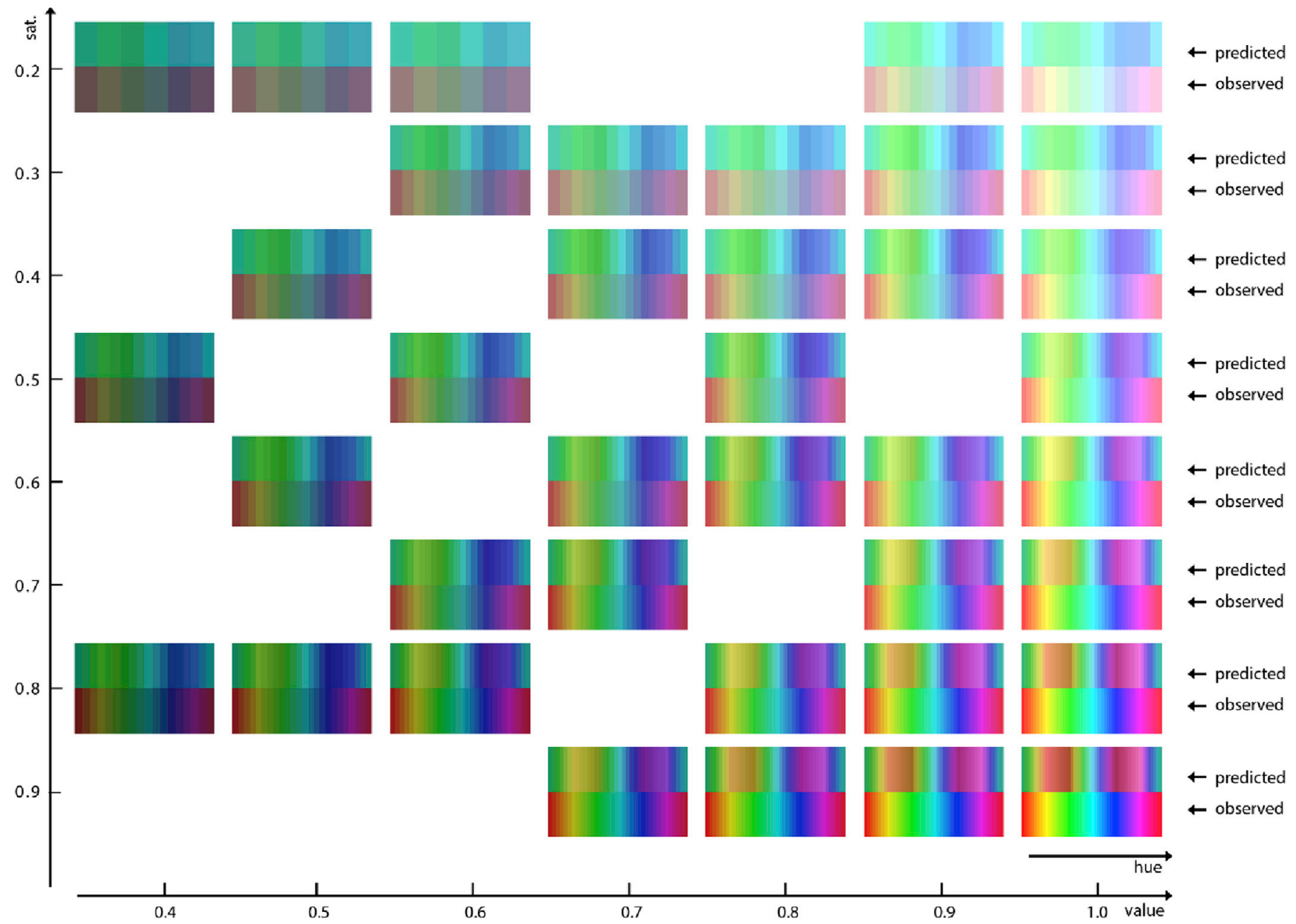

Fig. 5. Results from fitting the RGB-based encoding model on the HSV color stimuli. Each mini-panel shows observed and predicted colors for a specific saturation and value combination. Within each panel, bottom row shows the sequence of HSV stimuli (i.e., 'ground truth' or 'observed colors'), and the top row shows the predicted color from the RGB encoding model.

value. Within each panel, the bottom half shows the actual color and the top half shows the predicted color based on the RGB encoding model. The model fares well in predicting the value and the saturation of the color. However, while overall it captures the hue progression, there are significant deviations (e.g. failing to predict red colors, overpredicting yellow-brown colors, and underpredicting blue colors). These deviations occur because the model is trying to impose a linear transformation on non-linearly related representational spaces. In summary, a high predictive accuracy is not by itself sufficient to conclude that an encoding model reflects the nature of the underlying neural representation.

\section{Discussion}

Encoding models (Haxby et al., 2014; Naselaris et al., 2011) are a powerful new tool for studying how the constituent features of stimuli relate to the neural activation patterns elicited by these stimuli. They represent a significant methodological advance over more traditional MVPA methods because they allow us to predict neural activation for novel items and because they can be used to decode the identity of such items from neural data alone. While this is an impressive feat and an incredibly useful tool, we have to be cautious in interpreting what such successes mean for our understanding of the representational system of the brain. Both theorists (e.g., Haxby et al., 2014; Naselaris and Kay, 2015; Naselaris et al., 2011; Norman et al., 2006; Tong and Pratte, 2012) and practitioners (e.g. Fernandino et al., 2016; Kay et al., 2008; Mitchell et al., 2008; Santoro et al., 2014) have suggested that we can infer that the brain uses a certain set of features to encode information, if we can successfully predict the activity of novel items from such features. However, as we have argued here, this inference is not formally valid. Successful prediction might be the result of a systematic relationship between the representational system of the brain and the stimulus feature set, even if those utilize different representational schemes.

\section{Representational equivalence - is it in the eye of the beholder?}

How do we know whether two representational systems are truly different? It could be argued that in our first example, both binary and decimal number systems share many properties, and that they are merely different implementations of the same fundamental representation. For example, both systems use the position of a digit to encode its magnitude, and as a result, all arithmetic procedures that can be performed with decimal numbers can be applied to binary numbers as well. Despite these similarities, the transformation required to get the decimal from the binary representation of a number is non-linear. Linearity is important - it has been argued that two representational spaces could be considered equivalent only if there exists a linear transformation from one to the other (Naselaris et al., 2011). Linear transformations rotate multidimensional spaces, but do not change the nature of the representation, only the interpretation of the dimension. However, when the transformation required is non-linear, then the geometry of the underlying representational spaces is different (Kriegeskorte and Kievit, 2013).

We further propose that the key issue in determining whether two 
representational system are equivalent is whether you can establish a one-to-one mapping relation between features at different levels of representation in each system. For example, if you substitute each decimal digit with a unique letter, the resulting system would appear to be very different from the decimal system only on the surface - the relation between multi-digit numbers and their features would be the same in both cases. ${ }^{7}$ In contrast, decimal and binary features have a qualitatively different relation to the numbers they represent. Despite this, binary representations can be predicted based on decimal features, illustrating the inferential problem of encoding models we address here.

Simulation 2 allowed us to illustrate this point more clearly by directly visualizing the differences in the geometries of RGB and HSV color spaces. While both color models use 3 dimensions to represent colors, these dimensions have distinct interpretations and can be related by a systematic non-linear transformation. The RGB color model is an additive mixture space, in which any color is produced by mixing the three primary colors in different proportions. In contrast, the HSV model represents colors by specifying their hue (e.g., red), their saturation (i.e., the intensity of the pigment), and their value (i.e., the darkness of the pigment). These three-dimensional representational spaces have different geometries, such that the relative distances between colors are not identical. Yet, training an encoding model to predict the HSV representation of novel colors based on the relationship between the RGB and HSV features of other colors, was highly accurate on average.

\section{Where to go from here?}

\section{Model comparison}

An important question that naturally arises from the caveats we discussed is how one can maximize confidence in the outcome of a forward encoding model approach, or conversely, guard oneself against unjustified inferences. As others have noted, it is crucial to compare the performance of several possible encoding models (Haxby et al., 2014; Naselaris et al., 2011). If one model leads to a higher prediction accuracy than another, that is evidence that its features capture the variance of the neural representations better than the alternative model. However, it is not sufficient to use a "baseline model" that is unrelated to the domain of interest (i.e., comparing a semantic feature model to a low-level visual word form model as exemplified by Fernandino et al., 2016). Instead, one or several alternative representational models should be tested that are derived from competing theories (i.e., semantic model A vs. semantic model B). To illustrate, an elegant comparison of a sensory-based vs. non-sensory-based semantic model was achieved by Anderson et al. (2015). These authors contrasted a visual model with a word co-occurrence model to investigate which brain regions represent modality-specific visual features, and which do not (using differential correlation in RSA rather than an encoding model).

The relative superiority of a particular model at predicting activation patterns in a brain region makes it more likely that the brain is using the representational scheme of the better performing model rather than the alternative. However, it is important to keep in mind that such comparisons only provide evidence for the relative likelihood of each model, but, due to the limitations discussed above, still do not allow us to infer that the winning model is the "true" model (Palminteri et al., 2017). Additionally, when fitting these models, researchers need to account for differences in the number of parameters in each model and the risk of overfitting.

\section{Absolute model performance}

For that reason, besides the assessment of relative model performance based on model comparison, a second crucial step is to evaluate absolute

\footnotetext{
${ }^{7}$ In fact, because of that linear one-to-one relationship, replicating our simulation with these two examples leads to perfect prediction accuracy; compare that to the 0.7 prediction accuracy for the decimal-to-binary model.
}

prediction performance. In particular, the observed prediction accuracy can be compared to the "noise ceiling", or to the "upper limit of prediction accuracy" (Naselaris et al., 2011), reflecting the maximal performance that can be feasibly achieved given the noise present in the signal. The gap between the two can be thought of as the variance that is not explained by the current model, which should motivate and guide the search for an improved or alternative version of the model. Until such maximal performance is obtained, we should be careful in making strong representational inferences about the brain from the currently available analytic methods. It is important to note that assessing absolute model performance should not be done in isolation, but rather in combination with model comparison as a given model may in principle reach the noise ceiling even if a more appropriate model exists.

Beyond the prediction accuracy: visualizing and comparing the organization of predicted and observed neural activation patterns

It is important to note that even high decoding or predictive accuracy on its own is insufficient to establish representational equivalence. Despite the fact that in Simulation 2 our RGB encoding model predicted the HSV representation with an $82 \%$ rank accuracy, a more careful inspection of the actual and the predicted HSV colors revealed that there are significant deviations in the model predictions. This is because the regression model is attempting to establish a linear rotation between the non-linearly related RGB and HSV spaces.

This result should make us wary of making conclusions of representational equivalence based on a single accuracy value, as is the practice in some published studies (e.g. Fernandino et al., 2016). Rather, it is important to examine the actual correspondence of the representations with additional RSA methods or dimensionality reduction and visualization techniques (e.g., Brouwer and Heeger, 2009; Foster et al., 2015). For example, in studying color representation in the early visual cortex, Brouwer and Heeger (2009) used principal components analysis (PCA) on the predicted voxel activation patterns and showed that the two main components present in the signal corresponded well to the actual organization of the colors in the color space they were using. Similarly, Foster et al. (2015), who attempted to decode the orientation of lines that were held in visual working memory, showed that activity in the hypothesized angular channels, on which their encoding model was based, follows a graded pattern that peaks at their preferred orientation. In summary, we believe it is crucial to carefully examine the model predictions. If the encoding model reflects the actual representational space, there should be no systematicity in the errors it makes, and the relations between the predicted activation patterns should resemble the relations and the organization of the observed activation patterns.

\section{Attentional modulation}

Ultimately, many of these inferential caveats exist because fMRI data is correlational. Comparing alternative models and evaluating absolute prediction performance might eventually converge on the true underlying feature model, but this is not guaranteed. We propose that an even better way to test representational hypotheses might be to experimentally manipulate the hypothesized representational dimensions. For example, one could prime participants to weight or attend to some features of the stimuli more than others. This type of attentional modulation decreases noise in the correlated activity between individual neurons, which enhances population coding (Downer et al., 2015), and it also increases selectivity of individual neurons for task-relevant stimulus features (Sigala and Logothetis, 2002). Thus, if orienting attention to some features of the stimuli improves the ability of an encoding model based on those features to predict neural activity, this would constitute much stronger evidence for the viability of the model.

Relatively little work exists to evaluate this proposal with fMRI, although three recent studies present promising results with an attentional modulation method that could eventually be extended to encoding model analyses as well. Çukur et al. (2013) showed that when participants were searching for people or for vehicles in movie clips, individual 


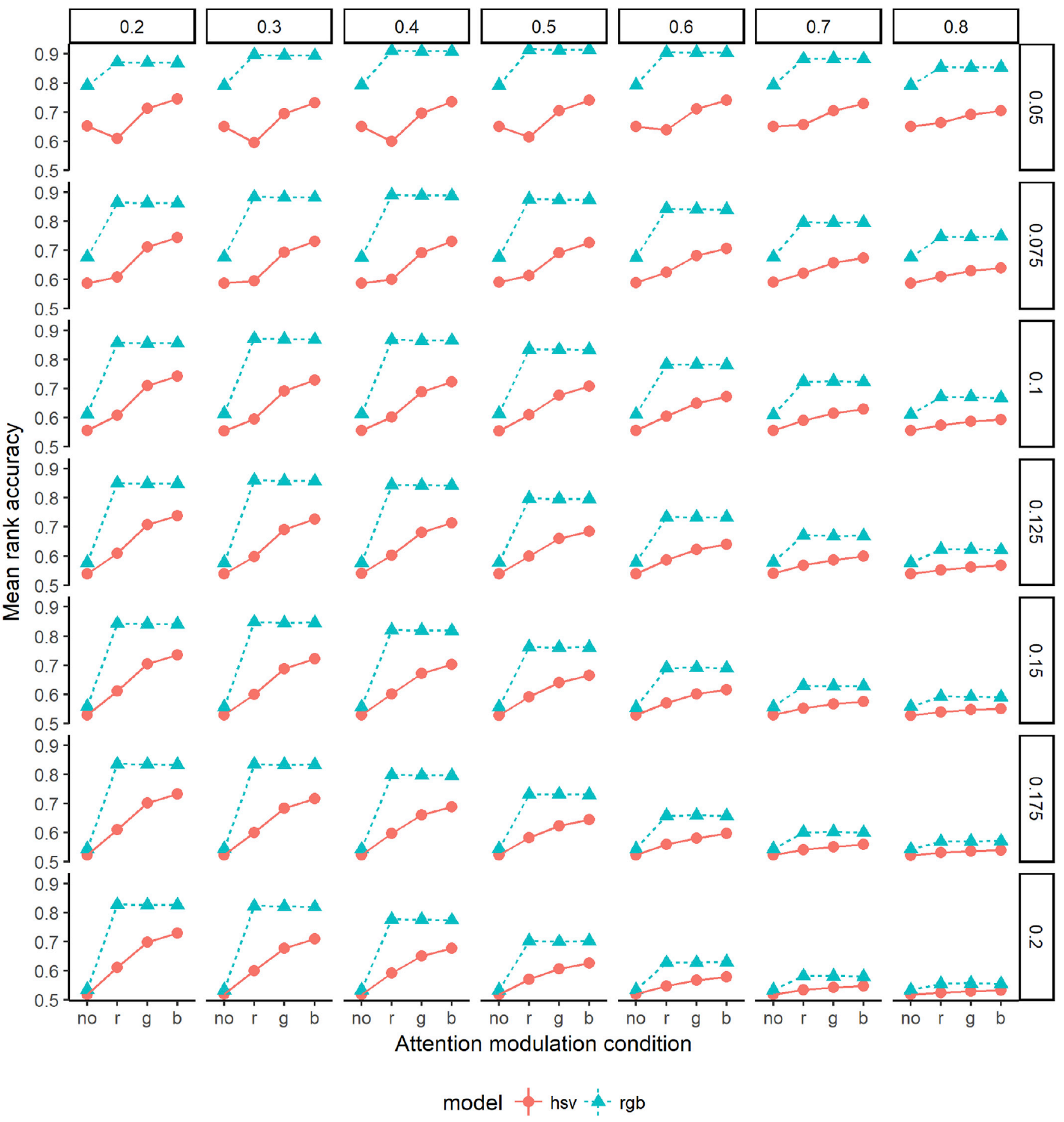

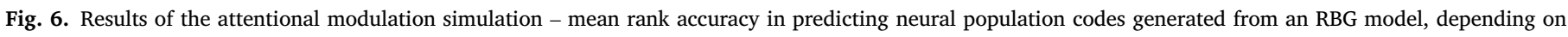

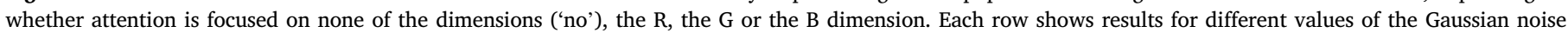

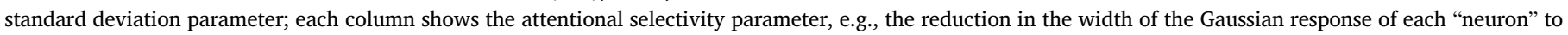
the attended dimension.

voxels became more attuned to the attended category. This occurred regardless of whether the target category was actually present in a scene. Importantly, this tuning shift was progressively stronger in higher-order visual areas compared to early retinotopic visual areas. Thus, attentional tuning likely reflects the fact that some aspect of the attended information is being represented in a specific ROI. Relatedly, Braunlich and Love
(2018), showed that when one feature of artificial category stimuli becomes more relevant for a categorization task, the ability to predict the presence of that feature increased. Additionally, they demonstrated that predictive accuracy correlated with attentional modulation parameters derived by cognitive models of conceptual representation.

Even more relevant is a recent study by Nastase et al., (2017), who 
extended this approach to representational similarity analysis. Participants saw a sequence of short clips with animals performing certain behaviors, and they had to respond to each clip in one of two ways. In one condition, the task was to say whether the animal in the current clip is from the same taxonomic category as the animal in the previous clip. In the other condition, participants had to respond whether the animal was performing the same behavior as the animal in the previous clip. The authors derived two representational dissimilarity matrices for the stimuli - one based on the taxonomic dissimilarity, and one based on the dissimilarity in the behavior that the animals performed. They calculated the correlation of these two representational dissimilarity matrix (RDM) with an RDM derived from the neural signal. When participants attended to the taxonomic category of the animals, the correlation between the neural and the taxonomic RDM increased. In contrast, attending to the animal's behavior increased the correlation of the neural RDM with the behavioral RDM.

Perhaps the most relevant finding of the study is that even though there was a significant correlation between the model RDMs and the neural RDMs in several regions, attentional modulation did not increase the correlation in all of them. For example, while the behavioral model correlated with neural activity in both the early visual cortex, and in postcentral parietal regions involved in motion perception, attending to the behavior of the animals increased the correlation only in the latter regions. Thus, on the basis of the overall correlation one might have concluded that behavior is represented even in early visual cortex; however, the lack of the attentional modulation in those areas indicates the overall correlation might have been driven by some visual information that is simply confounded with the animal's behavior. Similarly, taxonomic information correlated with the early visual cortex and the ventrotemporal cortex, but attending to taxonomy increased the correlation only in the latter. Nevertheless, one caveat to this proposal is that some brain areas might not be susceptible to attentional modulation. For that reason, it is important to note that while the presence of attentional modulation increases support for the model, the absence of such does not necessarily reduce it, and such inferences should be made with care.

Overall, these results provide some indication that modulating attention to specific features of the stimuli can be beneficial in determining whether successful prediction reflects the nature of the neural representation, or whether it is picking up systematically related information instead. We expect that this technique can be effectively extended to encoding models as well, as illustrated in Simulation 3.

\section{Simulation 3: attentional modulation}

One potential caveat to our proposal is that attentional modulation might increase performance even for incorrect models, if attention is focused on features that are highly correlated between the models. It seems natural to suggest that the extent to which attentional modulation will allow discrimination between models depends inversely on the degree to which that the attended dimension is correlated in the two models.

To test this intuition, we performed a third simulation, in which we extended our color prediction example. As we discussed above, one of the consequences of attentional modulation is the increased sensitivity of neurons to their preferred stimulus. In order to simulate attentional modulation, this time we simulated neural data from the RGB space in a different way. We assumed that each dimension is represented by a population code of five units, where each unit had a different preferred value on that dimension and its activity followed a Gaussian distribution around that preferred value (similarly to Foster et al., 2015). The preferred values were equally spaced from 0 to 1 (i.e., $0.1,0.3,0.5,0.7$, 0.9 ), and the default standard deviation of the response was 1 in the absence of attentional modulation. We additionally added Gaussian noise to the activity of each neuron, because prediction was nearly perfect for the correct RGB model.

To simulate increased selectivity due to attentional modulation, we reduced the width (i.e. the standard deviation) of the Gaussian response function for units that coded the attended dimension, and we increased the maximal response. The final response vector had 15 units, 5 for each of the three dimensions, and we compared how successful the RGB generating model and the alternative incorrect HSV model were in predicting the neural responses depending on four conditions - no attentional modulation, attending to the $\mathrm{R}$, to the $\mathrm{G}$ or to the $\mathrm{B}$ dimension. ${ }^{8}$ The R dimension was the least correlated on average with the HSV model $(r=0.24)$, followed by the $\mathrm{G}(r=0.38)$ and the $\mathrm{B}(\mathrm{r}=0.41)$.

We expected that for the true RGB model, the increase in predictive accuracy would be the same regardless of which dimension attention was focused on. However, for the incorrect HSV model, we expected that the increase will be proportional to how strongly the attended dimension was correlated with the model. We ran the simulation for a range of noise and selectivity parameters, and the results are presented in Fig. 6. Our predictions were confirmed across all parameter values. In summary, a predictive accuracy increase due to attentional modulation should increase trust in the model to the degree to which 1) focusing on the different dimension produces equivalent increases and 2) the attended dimension is not correlated with alternative models. This further suggests that attentional modulation might help identify which features of the model are more likely to capture a feature of the underlying representation.

\section{Final remarks}

Many of the points we have raised here are not specific to encoding models, but can also be leveled against any other method currently in use for understanding neural representations. The reason why we focused here on encoding models is that they are becoming increasingly popular, and we believe it is important to highlight their limitations. The speed at which multivariate methods have developed since Haxby et al. (2001) seminal study on multivariate analyses illustrates the importance of these methods to the neuroscientific community. Encoding models have many advantages over more traditional MVPA techniques, such as the ability to decode or predict activity for items that the model has not been trained on. However, because encoding models are so powerful, it is important to understand what inferences we can and cannot draw from them. There is little doubt that successful prediction or decoding in a certain brain area tells us a lot about where in the brain the information might be represented. The question of how those representations are organized, albeit, is more difficult and cannot be answered only on the basis of a single significant prediction value. Our hope with this commentary is not to downplay the important role of these methods, but to further the discussion about what we can and cannot learn by using them.

\section{Author contributions}

Conceptualization, V.P and M.O; Formal Analysis,V.P; Writing Original Draft, V.P. and M.O.; Writing - Review \& Editing, V.P., M.O. and C.T; Visualization, M.O. and V.P.

\section{Declarations of interest}

None.

\section{Acknowledgements}

We are grateful to Marc Coutanche, Timothy Verstynen and Brian MacWhinney for comments on a previous version of this draft. This research did not receive any specific grant from funding agencies in the public, commercial, or not-for-profit sectors.

\footnotetext{
8 This would be analogous to asking participants to 1) discriminate between colors; to respond which color is more 2) red, 3) blue or 4) green.
} 


\section{References}

Anderson, A.J., Bruni, E., Lopopolo, A., Poesio, M., Baroni, M., 2015. Reading visually embodied meaning from the brain: visually grounded computational models decode visual-object mental imagery induced by written text. NeuroImage 120, 309-322.

Binder, J.R., Desai, R.H., Graves, W.W., Conant, L.L., 2009. Where is the semantic System? A critical review and meta-analysis of 120 functional neuroimaging studies. Cereb. Cortex 19 (12), 2767-2796.

Bonnici, H.M., Kumaran, D., Chadwick, M.J., Weiskopf, N., Hassabis, D., Maguire, E.A., 2012. Decoding representations of scenes in the medial temporal lobes. Hippocampus 22 (5), 1143-1153.

Braunlich, K., Love, B.C., 2018, February 14. Occipitotemporal Representations Reflect Individual Differences in Conceptual Knowledge bioRxiv, 264895. Retrieved from. https://www.biorxiv.org/content/early/2018/02/14/264895. https://doi.org/10. $1101 / 264895$.

Brouwer, G.J., Heeger, D.J., 2009. Decoding and reconstructing color from responses in human visual cortex. J. Neurosci. 29 (44), 13992-14003.

Carlson, T.A., 2014. Orientation decoding in human visual cortex: new insights from an unbiased perspective. J. Neurosci. 34 (24), 8373-8383.

Casey, M., Thompson, J., Kang, O., Raizada, R., Wheatley, T., 2012. Population Codes Representing Musical Timbre for High-level fMRI Categorization of Music Genres. In Machine Learning and Interpretation in Neuroimaging. Springer Berlin Heidelberg, pp. 34-41.

Correia, J., Formisano, E., Valente, G., Hausfeld, L., Jansma, B., Bonte, M., 2014. Brainbased translation: fMRI decoding of spoken words in bilinguals reveals languageindependent semantic representations in anterior temporal lobe. J. Neurosci. 34 (1), 332-338.

Coutanche, M.N., 2013. Distinguishing multi-voxel patterns and mean activation: why, how, and what does it tell us? Cognit, Affect. Behav. Neurosci. 13 (3), 667-673.

Coutanche, M.N., Thompson-Schill, S.L., 2015. Creating concepts from converging features in human cortex. Cereb. Cortex 25 (9), 2584-2593.

Çukur, T., Nishimoto, S., Huth, A.G., Gallant, J.L., 2013. Attention during natural vision warps semantic representation across the human brain. Nat. Neurosci. 16, 763-770.

Davis, T., Poldrack, R.A., 2013. Measuring neural representations with fMRI: practices and pitfalls. Ann. N. Y. Acad. Sci. 1296 (1), 108-134.

Downer, J.D., Niwa, M., Sutter, M.L., 2015. Task engagement selectively modulates neural correlations in primary auditory cortex. J. Neurosci. 35 (19), 7565-7574.

Ewers, M., Sperling, R.A., Klunk, W.E., Weiner, M.W., Hampel, H., 2011. Neuroimaging markers for the prediction and early diagnosis of Alzheimer's disease dementia. Trends Neurosci. 34 (8), 430-442.

Farah, M.J., McClelland, J.L., 1991. A computational model of semantic memory impairment: modality specificity and emergent category specificity. J. Exp. Psychol. General 120 (4), 339.

Fernandino, L., Humphries, C.J., Conant, L.L., Seidenberg, M.S., Binder, J.R., 2016. Heteromodal cortical areas encode sensory-motor features of word meaning. J. Neurosci. 36 (38), 9763-9769.

Fodor, J.A., Pylyshyn, Z.W., 1988. Connectionism and cognitive architecture: a critical analysis. Cognition 28, 3-71.

Ford, A., Roberts, A., 1998. Color Space Conversions. Retrieved from. http://www. poynton.com/PDFs/coloureq.pdf.

Foster, J.J., Sutterer, D.W., Serences, J.T., Vogel, E.K., Awh, E., 2015. The topography of alpha-band activity tracks the content of spatial working memory. J. Neurophysiol. 115 (1), 168-177.

Freeman, J., Brouwer, G.J., Heeger, D.J., Merriam, E.P., 2011. Orientation decoding depends on maps, not columns. J. Neurosci. (13), 4792-4804.
Haxby, J.V., Connolly, A.C., Guntupalli, J.S., 2014. Decoding neural representational spaces using multivariate pattern analysis. Annu. Rev. Neurosci. 37 (1), 435-456.

Haxby, J.V., Gobbini, M.I., Furey, M.L., Ishai, A., Schouten, J.L., Pietrini, P., 2001. Distributed and overlapping representations of faces and objects in ventral temporal cortex. Science 293 (5539), 2425-2430.

Hebart, M.N., Baker, C.I., 2017. Deconstructing multivariate decoding for the study of brain function. NeuroImage.

Kamitani, Y., Tong, F., 2005. Decoding the visual and subjective contents of the human brain. Nat. Neurosci. 8 (5), 679.

Kay, K.N., Naselaris, T., Prenger, R.J., Gallant, J.L., 2008. Identifying natural images from human brain activity. Nature 452 (7185), 352-355.

Kriegeskorte, N., Kievit, R.A., 2013. Representational geometry: integrating cognition, computation, and the brain. Trends Cognit Sci. 17 (8), 401-412.

Lee, H., Kuhl, B.A., 2016. Reconstructing perceived and retrieved faces from activity patterns in lateral parietal cortex. J. Neurosci. 36 (22), 6069-6082.

Lotte, F., Congedo, M., Lécuyer, A., Lamarche, F., Arnaldi, B., 2007. A review of classification algorithms for EEG-based brain-computer interfaces. J. neural Eng. 4 (2), R1.

Mahon, B.Z., 2015. The burden of embodied cognition. Can. J. Exp. Psychol. 69 (2), 172-178.

Mitchell, T.M., Shinkareva, S.V., Carlson, A., Chang, K.-M., Malave, V.L., Mason, R.A., Just, M.A., 2008. Predicting human brain activity associated with the meanings of nouns. Science 320 (5880), 1191-1195.

Monaghan, P., Shillcock, R.C., Christiansen, M.H., Kirby, S., 2014. How arbitrary is language? Phil. Trans. R. Soc. B 369 (1651), 20130299.

Naselaris, T., Kay, K.N., 2015. Resolving ambiguities of MVPA using explicit models of representation. Trends Cognit Sci. 19 (10), 551-554.

Naselaris, T., Kay, K.N., Nishimoto, S., Gallant, J.L., 2011. Encoding and decoding in fMRI. NeuroImage 56 (2), 400-410.

Nastase, S.A., et al., 2017. Attention selectively reshapes the geometry of distributed semantic representation. Cerebr. Cortex 1-15. https://doi.org/10.1093/cercor/ bhx138.

Norman, K., Polyn, S., Detre, G., Haxby, J., 2006. Beyond mind-reading: multi-voxel pattern analysis of fMRI data. Trends Cognit Sci. 10 (9), 424-430.

Palminteri, S., Wyart, V., Koechlin, E., 2017. The importance of falsification in computational cognitive modeling. Trends Cognit Sci. Advance Online Publication.

Poldrack, R., 2006. Can cognitive processes be inferred from neuroimaging data? Trends Cognit Sci. 10 (2), 59-63.

Poldrack, R.A., 2011. Inferring mental states from neuroimaging data: from reverse inference to large-scale decoding. Neuron 72 (5), 692-697.

Ritchie, J.B., Kaplan, D., Klein, C., 2017, April 15. Decoding The Brain: Neural Representation And The Limits Of Multivariate Pattern Analysis In Cognitive Neuroscience bioRxiv, 127233. Retrieved from biorxiv.org/content/early/2017/04/ 15/127233. https://doi.org/10.1101/127233.

Santoro, R., Moerel, M., Martino, F.D., Goebel, R., Ugurbil, K., Yacoub, E., Formisano, E., 2014. Encoding of natural sounds at multiple spectral and temporal resolutions in the human auditory cortex. PLOS Comput. Biol. 10 (1), e1003412.

Sigala, N., Logothetis, N.K., 2002. Visual categorization shapes feature selectivity in the primate temporal cortex. Nature 415 (6869), 318-320.

Tong, F., Pratte, M., 2012. Decoding patterns of human brain activity. Annu. Rev. Psychol. 63 (1), 483-509.

Wagner, A.D., Schacter, D.L., Rotte, M., Koutstaal, W., Maril, A., Dale, A.M., Buckner, R.L., 1998. Building memories: remembering and forgetting of verbal experiences as predicted by brain activity. Science 281 (5380), 1188-1191. 